\title{
Fractional free space, fractional lenses, and fractional imaging systems
}

\author{
Uygar Sümbül and Haldun M. Ozaktas \\ Department of Electrical Engineering, Bilkent University, TR-06533 Bilkent, Ankara, Turkey
}

Received March 18, 2003, revised manuscript received June 25, 2003; accepted July 18, 2003

\begin{abstract}
Continuum extensions of common dual pairs of operators are presented and consolidated, based on the fractional Fourier transform. In particular, the fractional chirp multiplication, fractional chirp convolution, and fractional scaling operators are defined and expressed in terms of their common nonfractional special cases, revealing precisely how they are interpolations of their conventional counterparts. Optical realizations of these operators are possible with use of common physical components. These three operators can be interpreted as fractional lenses, fractional free space, and fractional imaging systems, respectively. Any optical system consisting of an arbitrary concatenation of sections of free space and thin lenses can be interpreted as a fractional imaging system with spherical reference surfaces. As a special case, a system departing from the classical single-lens imaging condition can be interpreted as a fractional imaging system. () 2003 Optical Society of America
\end{abstract}

OCIS codes: $070.0070,070.2580,080.0080,080.2720$.

\section{INTRODUCTION}

The fractional Fourier transform, which is a generalization of the ordinary Fourier transform, has received considerable interest over the past decade and has found many applications in optics and signal processing. ${ }^{1-24}$ Of particular interest from an optics perspective is the observation that as light propagates, its amplitude distribution evolves through fractional Fourier transforms of increasing orders. This observation is based on a relationship between the fractional Fourier transform and the Fresnel transform with the fractional order being related to and increasing with the distance of propagation. ${ }^{25}$ With use of this result, it is possible to analyze a wide family of optical systems. ${ }^{1,26}$ An important concept is that of fractional Fourier domains, which are generalizations of the conventional space and frequency domains. ${ }^{27,28}$ This continuum of domains provides a continuous transition between the space and frequency domains.

Several pairs of operators are known to be Fourier duals (or conjugates). (For a discussion of such duals in an optics context, see Refs. 29 and 30.) Coordinate multiplication/differentiation and phase-shift/translation operators are just two common dual pairs. In this paper we will introduce and consolidate several continuums of operators indexed by the fractional order parameter $a$, whose members are associated with the ath fractional Fourier domain and which likewise provide a continuous transition between common dual operator pairs.

The operators that we deal with in this paper perform the same actions in fractional domains as their conventional counterparts perform in the space domain. We will also discuss the optical implementation of these operators in terms of their conventional counterparts.

The ath-order fractional Fourier transform $f_{a}(u)$ $=\left\{\mathcal{F}^{a} f\right\}(u)$ of the function $f(u)$ is defined for $0<|a|$ $<2$ as

$$
\begin{aligned}
\mathcal{F}^{a}[f(u)] \equiv & \left\{\mathcal{F}^{a} f\right\}(u) \equiv \int_{-\infty}^{\infty} K_{a}\left(u, u^{\prime}\right) f\left(u^{\prime}\right) \mathrm{d} u^{\prime}, \\
K_{a}\left(u, u^{\prime}\right) \equiv & \frac{\exp \{-i[\pi \operatorname{sgn}(\alpha) / 4-\alpha / 2]\}}{|\sin \alpha|^{1 / 2}} \\
& \times \exp \left[i \pi \left(\cot \alpha u^{2}-2 \csc \alpha u u^{\prime}\right.\right. \\
& \left.\left.+\cot \alpha u^{\prime 2}\right)\right]
\end{aligned}
$$

where

$$
\alpha \equiv a \pi / 2 \text {. }
$$

The definition may be extended outside the interval $(-2,2)$ through $\mathcal{F}^{4 j+a} f=\mathcal{F}^{a} f$ for any integer $j$. Moreover, $\mathcal{F}^{4 j}$ and $\mathcal{F}^{4 j+1}$ correspond to the identity operator $\mathcal{I}$ and the ordinary Fourier transform operator $\mathcal{F}$, respectively. The transform is a linear operator, and it is additive in index: $\mathcal{F}^{a_{1}} \mathcal{F}^{a_{2}} f=\mathcal{F}^{a_{1}+a_{2}} f$. Other properties are given in Ref. 1 .

It will also be useful to review the three-parameter group of linear integral transforms known as linear canonical transforms, of which the fractional Fourier transform is a special case. The linear canonical transform of $f(u)$, denoted $f_{\mathbf{M}}(u)=\left\{\mathcal{C}_{\mathbf{M}} f\right\}(u)$, is defined as ${ }^{31}$

$$
\begin{aligned}
\left\{\mathcal{C}_{\mathbf{M}} f\right\}(u) & =\int C_{\mathbf{M}}\left(u, u^{\prime}\right) f\left(u^{\prime}\right) \mathrm{d} u^{\prime}, \\
C_{\mathbf{M}}\left(u, u^{\prime}\right) & =A_{\mathbf{M}} \exp \left[i \pi\left(\alpha u^{2}-2 \beta u u^{\prime}+\gamma u^{\prime 2}\right)\right],
\end{aligned}
$$

where $A_{\mathbf{M}}=\sqrt{\beta} \exp (-i \pi / 4)$. Here $\mathcal{C}_{\mathbf{M}}$ is the linear canonical transform operator and $\mathbf{M}$ represents the three real parameters $\alpha, \beta, \gamma$. It is convenient to represent $\mathbf{M}$ in matrix form:

$$
\mathbf{M} \equiv\left[\begin{array}{cc}
\gamma / \beta & 1 / \beta \\
-\beta+\alpha \gamma / \beta & \alpha / \beta
\end{array}\right] \equiv\left[\begin{array}{ll}
A & B \\
C & D
\end{array}\right] .
$$


The reason we define $\mathbf{M}$ in this manner is that the matrix corresponding to the composition of two systems is the matrix product of the matrices of the corresponding individual systems. Moreover, the matrix of the inverse of a transform corresponds to the inverse of the original transform's matrix.

The fractional Fourier transform operator is a linear canonical transform whose matrix is the rotation matrix

$$
\mathcal{F}^{a}=\exp (\text { ia } \pi / 4) \mathcal{C}_{\mathbf{M}},
$$

where

$$
\mathbf{M}=\left[\begin{array}{cc}
\cos \alpha & \sin \alpha \\
-\sin \alpha & \cos \alpha
\end{array}\right] .
$$

We note that some of the developments and results of this paper can be seen as special cases of corresponding results for linear canonical transforms. In some cases this is merely a matter of substituting the matrix parameters given in Eq. (6). For such more general results, we refer the reader to general studies on linear canonical transforms and operator methods. ${ }^{32-47}$

The effect of ath-order fractional Fourier transformation on the Wigner distribution of a signal is to rotate the Wigner distribution by an angle $\alpha .^{27,28,48}$ Hence the mathematical relation between the Wigner distribution of a function and the distribution of its fractional Fourier transform is as follows:

$$
W_{f_{a}}(u, \mu)=W_{f}(u \cos \alpha-\mu \sin \alpha, u \sin \alpha+\mu \cos \alpha) .
$$

The Radon transform operator $\mathcal{R} \mathcal{D} \mathcal{N}_{\alpha}$, which takes the integral projection of the function $W_{f}(u, \mu)$ onto an axis making an angle $\alpha=a \pi / 2$ with the $u$ axis, can be used to restate the previous property in the following manner:

$$
\left\{\mathcal{R D N}_{\alpha}\left[W_{f}(u, \mu)\right]\right\}\left(u_{a}\right)=\left|f_{a}\left(u_{a}\right)\right|^{2} .
$$

Here the projection axis $u_{a}$ is referred to as the $a$ th fractional Fourier domain (Fig. 1). ${ }^{27,28}$ The space and frequency domains are merely special cases of the continuum of fractional Fourier domains.

It is important to distinguish between two distinct senses of the term "fractional" as it applies to operators. The first and more common is the sense in which we speak of the $a$ th fractional power of an operator $\mathcal{A}$, which we denote by $\mathcal{A}^{a}$. The fractional Fourier transform is indeed the $a$ th mathematical power of the ordinary Fourier transform in this sense. ${ }^{49}$

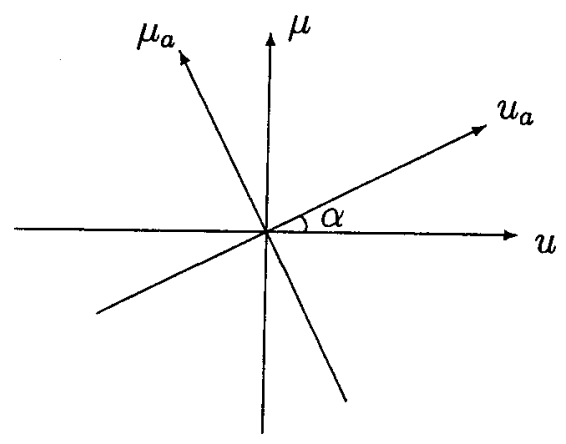

Fig. 1. The $a$ th fractional Fourier domain.
Second, we may speak of the operator that has the same effect on the $a$ th fractional Fourier transform $f_{a}\left(u_{a}\right)$ in the ath domain as the original operator has on the original function $f(u)$ in the space domain. To distinguish this operator associated with the ath fractional Fourier domain from the $a$ th power of $\mathcal{A}$, we will denote it by $\mathcal{A}_{a}$. From this definition it follows that

$$
\mathcal{A}_{a}=\mathcal{F}^{-a} \mathcal{A F}^{a} \text {. }
$$

Note that $\mathcal{A}$ and $\mathcal{A}_{a}$ are two different operators, whose representations in the 0 th and $a$ th domains, respectively, are identical. They are not different representations of the same operator.

It is this second kind of "fractional" operator that we will be dealing with in this paper. Thus when we speak of fractional free space or fractional lenses or fractional imaging systems, we will not be referring to fractional powers of an ordinary section of free space, lens, or imaging system. We will rather be referring to that system which has the same effect on a distribution of light represented in the fractional Fourier domain as its conventional counterpart would have in the space domain.

Indeed, the ath fractional power of a section of free space of length $d$ in the first sense is merely a section of free space of length ad. Likewise, the fractional power of a lens with focal length $f$ in the first sense is merely a lens with focal length $f / a$. It can be easily verified that these fractional powers satisfy the interpolation property at $a=0$ and $a=1$ and index additivity.

In the following sections we will first focus on coordinate multiplication/differentiation and phase-shifttranslation operator pairs and discuss their fractional counterparts. Section 4 deals with the fractional scaling operator, which will be seen to correspond to fractional imaging in an optics context. Section 5 deals with fractional chirp multiplication and convolution operators, which will be seen to correspond to fractional lenses and free space in an optics context. In Section 6 we will revisit the fractional scaling operator to discuss an important interpretation. The final section will discuss the importance of our results in an optical context.

\section{COORDINATE MULTIPLICATION AND DIFFERENTIATION OPERATORS}

We begin by defining the multiplication operator $\mathcal{U}$ and the differentiation operator $\mathcal{D}$ through their effects in the space domain:

$$
\begin{aligned}
& \{\mathcal{U} f\}(u)=u f(u), \\
& \{\mathcal{D} f\}(u)=(i 2 \pi)^{-1} \frac{\mathrm{d} f(u)}{\mathrm{d} u} .
\end{aligned}
$$

It can be easily shown that this pair forms a Fourier dual, which means that coordinate multiplication in the space domain corresponds to differentiation in the frequency domain and vice versa: The Fourier transform of $u f(u)$ is $(-i 2 \pi)^{-1} \mathrm{~d} F(\mu) / \mathrm{d} \mu$, and the Fourier transform of $(i 2 \pi)^{-1} \mathrm{~d} f(u) / \mathrm{d} u$ is $\mu F(\mu)$. A consequence is that $\mathcal{U}$ and $\mathcal{D}$ are related through the Fourier transform operator as follows: 


$$
\mathcal{D}=\mathcal{F}^{-1} \mathcal{U F}
$$

The fractional forms of these operators are defined so as to have the same functional effect in the $a$ th domain:

$$
\begin{aligned}
& \left\{\mathcal{U}_{a} f_{a}\right\}\left(u_{a}\right)=u_{a} f_{a}\left(u_{a}\right), \\
& \left\{\mathcal{D}_{a} f_{a}\right\}\left(u_{a}\right)=(i 2 \pi)^{-1} \frac{\mathrm{d} f_{a}\left(u_{a}\right)}{\mathrm{d} u_{a}} .
\end{aligned}
$$

From this definition we may obtain, generalizing Eq. (12) and consistent with the general form given in Eq. (9),

$$
\begin{aligned}
\mathcal{U}_{a} & =\mathcal{F}^{-a} \mathcal{U} \mathcal{F}^{a}, \\
\mathcal{D}_{a} & =\mathcal{F}^{-a} \mathcal{D} \mathcal{F}^{a},
\end{aligned}
$$

where

$$
\begin{array}{ll}
\mathcal{U}_{0}=\mathcal{U}, & \mathcal{U}_{1}=\mathcal{D}, \\
\mathcal{D}_{0}=\mathcal{D}, & \mathcal{U}_{-1}=-\mathcal{D}, \\
\mathcal{D}_{1}=-\mathcal{U}, & \mathcal{D}_{-1}=\mathcal{U} .
\end{array}
$$

It has been shown (for instance (see Refs. 27, 28, and 31), that these fractional operators can be expressed in terms of their integer counterparts as follows:

$$
\begin{aligned}
& \mathcal{U}_{a}=\cos \alpha \mathcal{U}+\sin \alpha \mathcal{D}, \\
& \mathcal{D}_{a}=-\sin \alpha \mathcal{U}+\cos \alpha \mathcal{D} .
\end{aligned}
$$

As the fractional-order parameter $a$ varies from 0 to 1 , the relative contributions of $\mathcal{U}$ and $\mathcal{D}$ to $\mathcal{U}_{a}$ and $\mathcal{D}_{a}$ are given by simple trigonometric factors. We also point out that these relations or their more general forms corresponding to more general linear canonical transforms follow immediately from an alternative definition of the fractional Fourier transform (Ref. 1, pp. 126-129) or linear canonical transforms ${ }^{31}$ in terms of $\mathcal{U}$ and $\mathcal{D}$.

\section{PHASE-SHIFT AND TRANSLATION OPERATORS}

The phase-shift operator $\mathcal{P} \mathcal{H}(\xi)$ and the translation operator $\mathcal{S H}(\xi)$ are defined as follows:

$$
\begin{aligned}
\mathcal{P} \mathcal{H}(\xi) & =\exp (i 2 \pi \xi \mathcal{U}), \\
\mathcal{S H}(\xi) & =\exp (i 2 \pi \xi \mathcal{D}) .
\end{aligned}
$$

These operators shift or translate signals in the space or frequency domain, respectively ${ }^{1}$ :

$$
\begin{aligned}
& \{\mathcal{P} \mathcal{H}(\xi) f\}(u)=\exp (i 2 \pi \xi u) f(u), \\
& \{\mathcal{S H}(\xi) f\}(u)=f(u+\xi) .
\end{aligned}
$$

Phase shifting and translation are Fourier duals of each other, which again means that phase shifting in the space domain corresponds to translation in the frequency domain and vice versa: The Fourier transform of $\exp (i 2 \pi \xi u) f(u)$ is $F(\mu-\xi)$, and the Fourier transform of $f(u-\xi)$ is $\exp (-i 2 \pi \xi \mu) F(\mu)$. Consequently, the two operators in question are related through

$$
\mathcal{S} \mathcal{H}(\xi)=\mathcal{F}^{-1} \mathcal{P} \mathcal{H}(\xi) \mathcal{F}
$$

which is of the same form as Eq. (12).

The fractional forms of these operators are defined $\operatorname{as}^{27,28}$

$$
\begin{aligned}
& \mathcal{P} \mathcal{H}_{a}(\xi)=\exp \left(i 2 \pi \xi \mathcal{U}_{a}\right) \\
& \mathcal{S} \mathcal{H}_{a}(\xi)=\exp \left(i 2 \pi \xi \mathcal{D}_{a}\right)
\end{aligned}
$$

where

$$
\begin{aligned}
\mathcal{P} \mathcal{H}_{0}(\xi) & =\mathcal{P} \mathcal{H}(\xi), & \mathcal{P} \mathcal{H}_{1}(\xi)=\mathcal{S H}(\xi), \\
\mathcal{P} \mathcal{H}_{-1}(\xi) & =\mathcal{S H}(-\xi), & \mathcal{S} \mathcal{H}_{0}(\xi)=\mathcal{S H}(\xi),
\end{aligned}
$$

$\mathcal{S} \mathcal{H}_{1}(\xi)=\mathcal{P} \mathcal{H}(-\xi), \quad \mathcal{S} \mathcal{H}_{-1}(\xi)=\mathcal{P} \mathcal{H}(\xi)$. These operators have the same functional effect in the $a$ th domain as their conventional counterparts have in the space domain:

$$
\begin{aligned}
& \left\{\mathcal{P} \mathcal{H}_{a}(\xi) f_{a}\right\}\left(u_{a}\right)=\exp \left(i 2 \pi \xi u_{a}\right) f_{a}\left(u_{a}\right), \\
& \left\{\mathcal{S} \mathcal{H}_{a}(\xi) f_{a}\right\}\left(u_{a}\right)=f_{a}\left(u_{a}+\xi\right) .
\end{aligned}
$$

By using the power series expansion of the fractional phase shift operator, we obtain

$$
\begin{aligned}
\mathcal{P} \mathcal{H}_{a}(\xi) & =\sum_{n=0}^{\infty} \frac{(i 2 \pi \xi)^{n}}{n !} \mathcal{U}_{a}{ }^{n} \\
& =\mathcal{F}^{-a} \sum_{n=0}^{\infty} \frac{(i 2 \pi \xi)^{n}}{n !} \mathcal{U}^{n} \mathcal{F}^{a} \\
& =\mathcal{F}^{-a} \mathcal{P} \mathcal{H}(\xi) \mathcal{F}^{a} .
\end{aligned}
$$

Similarly, it can be shown that the fractional translation operator satisfies

$$
\mathcal{S} \mathcal{H}_{a}(\xi)=\mathcal{F}^{-a} \mathcal{S} \mathcal{H}(\xi) \mathcal{F}^{a} .
$$

Equations (28) and (29) could have been taken as alternative definitions for the fractional phase-shift and translation operators [see Eq. (9)]. Let us substitute Eqs. (17) and (18) into the hyperdifferential forms given in Eqs. (24) and (25) and apply the well-known formula ${ }^{50}$

$$
\exp (\mathcal{A}) \exp (\mathcal{B})=\exp (\mathcal{A}+\mathcal{B}) \exp ([\mathcal{A}, \mathcal{B}] / 2),
$$

where both $\mathcal{A}$ and $\mathcal{B}$ commute with their commutator: $[\mathcal{A},[\mathcal{A}, \mathcal{B}]]=0$ and $[\mathcal{B},[\mathcal{A}, \mathcal{B}]]=0$. The commutator $[\mathcal{A}, \mathcal{B}]$ of $\mathcal{A}$ and $\mathcal{B}$ is defined as $[\mathcal{A}, \mathcal{B}]=\mathcal{A B}-\mathcal{B} \mathcal{A}$. This leads to the following equations expressing the operators in question in terms of their integer counterparts ${ }^{27,28}$ :

$$
\begin{aligned}
\mathcal{P} \mathcal{H}_{a}(\xi)= & \exp \left(i \pi \xi^{2} \sin \alpha \cos \alpha\right) \mathcal{P} \mathcal{H}(\xi \cos \alpha) \mathcal{S H}(\xi \sin \alpha), \\
\mathcal{S} \mathcal{H}_{a}(\xi)= & \exp \left(i \pi \xi^{2} \sin \alpha \cos \alpha\right) \mathcal{S} \mathcal{H}(\xi \cos \alpha) \\
& \times \mathcal{P} \mathcal{H}(-\xi \sin \alpha)
\end{aligned}
$$

As the fractional-order parameter $a$ varies from 0 to 1 , the relative contributions of $\mathcal{P} \mathcal{H}(\xi)$ and $\mathcal{S} \mathcal{H}(\xi)$ to $\mathcal{P} \mathcal{H}_{a}(\xi)$ and $\mathcal{S} \mathcal{H}_{a}(\xi)$ are given by simple trigonometric factors. It is interesting to compare these equations with Eqs. (17) and (18).

\section{SCALING OR MAGNIFICATION OPERATOR}

The scaling operator $\mathcal{M}(M)$ can be defined through the multiplication and differentiation operators in the following way $^{1}$ :

$$
\mathcal{M}(M)=\exp [-i \pi \ln M(\mathcal{U D}+\mathcal{D U})]
$$


where $M>0$. Its effect in the space domain is

$$
\{\mathcal{M}(M) f\}(u)=\sqrt{1 / M} f(u / M) .
$$

The scaling operator, which corresponds to magnified or demagnified imaging in optics, is its own dual in the sense that scaling in the space domain corresponds to descaling in the frequency domain: The Fourier transform of $\sqrt{1 / M} f(u / M)$ is $\sqrt{M} F(M \mu)$. Consequently,

$$
\mathcal{M}(M)=\mathcal{F}^{-1} \mathcal{M}(1 / M) \mathcal{F},
$$

which is a result of the following identity:

$$
\mathcal{U D}+\mathcal{D} \mathcal{U}=\mathcal{F}^{-1}[-(\mathcal{U D}+\mathcal{D} \mathcal{U})] \mathcal{F} .
$$

The scaling operator is a one-parameter subgroup of the group of linear canonical transforms with a $2 \times 2$ matrix:

$$
\mathbf{M}(M)=\left[\begin{array}{cc}
M & 0 \\
0 & 1 / M
\end{array}\right]=\left[\begin{array}{cc}
1 / M & 0 \\
0 & M
\end{array}\right]^{-1} .
$$

The fractional form of the scaling operator is again defined in the same manner:

$$
\mathcal{M}_{a}(M)=\exp \left[-i \pi \ln M\left(\mathcal{U}_{a} \mathcal{D}_{a}+\mathcal{D}_{a} \mathcal{U}_{a}\right)\right]
$$

where

$$
\mathcal{M}_{0}(M)=\mathcal{M}(M), \quad \mathcal{M}_{1}(M)=\mathcal{M}(1 / M),
$$

$\mathcal{M}_{-1}(M)=\mathcal{M}(1 / M)$. This operator has the same functional effect in the ath domain as its conventional counterpart has in the space domain:

$$
\left\{\mathcal{M}_{a}(M) f_{a}\right\}\left(u_{a}\right)=\sqrt{1 / M} f_{a}\left(u_{a} / M\right)
$$

From this definition we again obtain, in the form of Eq. (9),

$$
\mathcal{M}_{a}(M)=\mathcal{F}^{-a} \mathcal{M}(M) \mathcal{F}^{a} .
$$

Noting that $\mathcal{M}_{a}(M)$ is also a linear canonical transform, we use Eq. (39) to obtain its matrix as

$\mathbf{M}_{a}(M)$

$$
\begin{aligned}
& =\left[\begin{array}{cc}
\cos \alpha & -\sin \alpha \\
\sin \alpha & \cos \alpha
\end{array}\right]\left[\begin{array}{cc}
M & 0 \\
0 & 1 / M
\end{array}\right]\left[\begin{array}{cc}
\cos \alpha & \sin \alpha \\
-\sin \alpha & \cos \alpha
\end{array}\right] \\
& =\left[\begin{array}{cc}
M \cos ^{2} \alpha+\sin ^{2} \alpha / M & (M-1 / M) \sin \alpha \cos \alpha \\
(M-1 / M) \sin \alpha \cos \alpha & M \sin ^{2} \alpha+\cos ^{2} \alpha / M
\end{array}\right],
\end{aligned}
$$

where the phase factors from Eq. (5) cancel each other.

By using Eqs. (34) and (39) we can write

$$
\mathcal{M}_{a}(M)=\mathcal{F}^{-a} \mathcal{M}(M) \mathcal{F}^{a}=\mathcal{F}^{-1-a} \mathcal{M}(1 / M) \mathcal{F}^{1+a} .
$$

This result expresses the fractional scaling operator in terms of the ordinary scaling operator or its dual (which is also a scaling operator with reciprocal parameter). We can see that when $a=0, \mathcal{M}_{0}(M)=\mathcal{M}(M)$ and when $a$ $=1, \mathcal{M}_{1}(M)=\mathcal{M}(1 / M)$. As the fractional-order parameter $a$ varies from 0 to $1, \mathcal{M}_{a}(M)$ evolves from $\mathcal{M}(M)$ to $\mathcal{M}(1 / M)$. Also, compare this equation with Eqs. (17) and (18) and Eqs. (30) and (31).

\section{CHIRP MULTIPLICATION AND CHIRP CONVOLUTION}

The chirp multiplication operator $\mathcal{Q}(q)$ and the chirp convolution operator $\mathcal{R}(r)$ are defined as follows:

$$
\begin{aligned}
& \mathcal{Q}(q)=\exp \left(-i \pi q \mathcal{U}^{2}\right), \\
& \mathcal{R}(r)=\exp \left(-i \pi r \mathcal{D}^{2}\right) .
\end{aligned}
$$

Their effect in the space domain is given by

$$
\begin{aligned}
& \{\mathcal{Q}(q) f\}(u)=\exp \left(-i \pi q u^{2}\right) f(u), \\
& \{\mathcal{R}(r) f\}(u)=\exp (-i \pi / 4) \sqrt{1 / r} \exp \left(i \pi u^{2} / r\right) * f(u) .
\end{aligned}
$$

These two operators again form a Fourier dual pair, meaning that multiplying with a chirp function in the space domain corresponds to convolving with a chirp function in the frequency domain and vice versa. ${ }^{1}$ The chirp multiplication and chirp convolution operators are related through

$$
\mathcal{R}(r)=\mathcal{F}^{-1} \mathcal{Q}(r) \mathcal{F},
$$

which is again in the form of Eq. (9). The chirp multiplication operator describes the action of a thin lens on a field incident on it, and the chirp convolution operator describes the action of propagation through a section of free space in the Fresnel approximation. ${ }^{1}$

These chirp operators are one-parameter subgroups of the group of linear canonical transforms with $2 \times 2$ matrices:

$$
\begin{aligned}
& \mathbf{Q}(q)=\left[\begin{array}{cc}
1 & 0 \\
-q & 1
\end{array}\right]=\left[\begin{array}{ll}
1 & 0 \\
q & 1
\end{array}\right]^{-1}, \\
& \mathbf{R}(r)=\left[\begin{array}{ll}
1 & r \\
0 & 1
\end{array}\right]=\left[\begin{array}{cc}
1 & -r \\
0 & 1
\end{array}\right]^{-1} .
\end{aligned}
$$

The fractional forms of these operators are defined as

$$
\begin{aligned}
& \mathcal{Q}_{a}(q)=\exp \left(-i \pi q \mathcal{U}_{a}{ }^{2}\right), \\
& \mathcal{R}_{a}(r)=\exp \left(-i \pi r \mathcal{D}_{a}{ }^{2}\right),
\end{aligned}
$$

where

$$
\begin{array}{ll}
\mathcal{Q}_{0}(q)=\mathcal{Q}(q), & \mathcal{Q}_{1}(q)=\mathcal{R}(q), \\
\mathcal{Q}_{-1}(q)=\mathcal{R}(q), & \mathcal{R}_{0}(r)=\mathcal{R}(r), \\
\mathcal{R}_{1}(r)=\mathcal{Q}(r), & \mathcal{R}_{-1}(r)=\mathcal{Q}(r) .
\end{array}
$$

These operators have the same functional effect in the $a$ th domain as their conventional counterparts have in the space domain:

$$
\left\{\mathcal{Q}_{a}(q) f_{a}\right\}\left(u_{a}\right)=\exp \left(-i \pi q u_{a}^{2}\right) f_{a}\left(u_{a}\right),
$$

$\left\{\mathcal{R}_{a}(r) f_{a}\right\}\left(u_{a}\right)$

$$
=\exp (-i \pi / 4) \sqrt{1 / r} \exp \left(i \pi u_{a}{ }^{2} / r\right) * f_{a}\left(u_{a}\right) .
$$

It can again be shown through their power series expansion that these definitions imply

$$
\begin{aligned}
& \mathcal{Q}_{a}(q)=\mathcal{F}^{-a} \mathcal{Q}(q) \mathcal{F}^{a}, \\
& \mathcal{R}_{a}(r)=\mathcal{F}^{-a} \mathcal{R}(r) \mathcal{F}^{a} .
\end{aligned}
$$


Noting that $\mathcal{Q}_{a}(q)$ and $\mathcal{R}_{a}(r)$ are also linear canonical transforms, we can use Eq. (53) to obtain a matrix representation for $\mathcal{Q}_{a}(q)$ as

$$
\begin{aligned}
\mathbf{Q}_{a}(q) & =\left[\begin{array}{cc}
\cos \alpha & -\sin \alpha \\
\sin \alpha & \cos \alpha
\end{array}\right]\left[\begin{array}{cc}
1 & 0 \\
-q & 1
\end{array}\right]\left[\begin{array}{cc}
\cos \alpha & \sin \alpha \\
-\sin \alpha & \cos \alpha
\end{array}\right] \\
& =\left[\begin{array}{cc}
1+q \sin \alpha \cos \alpha & q \sin ^{2} \alpha \\
-q \cos ^{2} \alpha & 1-q \sin \alpha \cos \alpha
\end{array}\right],
\end{aligned}
$$

which can be written as

$$
\mathbf{Q}_{a}(q)=\left[\begin{array}{cc}
1 & -\tan \alpha \\
0 & 1
\end{array}\right]\left[\begin{array}{cc}
1 & 0 \\
-q \cos ^{2} \alpha & 1
\end{array}\right]\left[\begin{array}{cc}
1 & \tan \alpha \\
0 & 1
\end{array}\right] .
$$

Recognizing the matrices on the right-hand side as integer chirp operators enables us to express the fractional chirp multiplication operator $\mathcal{Q}_{a}(q)$ in terms of $\mathcal{Q}_{0}(q)$ and $\mathcal{R}_{0}(r)$ as

$$
\mathcal{Q}_{a}(q)=\mathcal{R}(-\tan \alpha) \mathcal{Q}\left(q \cos ^{2} \alpha\right) \mathcal{R}(\tan \alpha) .
$$

If we employ the same technique for the fractional chirp convolution operator $\mathcal{R}_{a}(r)$, we will arrive at an analogous result:

$$
\begin{aligned}
& \mathbf{R}_{a}(r)=\left[\begin{array}{cc}
1-r \sin \alpha \cos \alpha & r \cos ^{2} \alpha \\
-r \sin ^{2} \alpha & 1+r \sin \alpha \cos \alpha
\end{array}\right], \\
& \mathcal{R}_{a}(r)=\mathcal{R}(\cot \alpha) \mathcal{Q}\left(r \sin ^{2} \alpha\right) \mathcal{R}(-\cot \alpha) .
\end{aligned}
$$

Use of dual matrix decompositions [that is, with the lower and upper triangular matrices that appear in Eq. (56) interchanged], we can obtain two further equations as follows:

$$
\begin{aligned}
& \mathcal{Q}_{a}(q)=\mathcal{Q}(\cot \alpha) \mathcal{R}\left(q \sin ^{2} \alpha\right) \mathcal{Q}(-\cot \alpha), \\
& \mathcal{R}_{a}(r)=\mathcal{Q}(-\tan \alpha) \mathcal{R}\left(r \cos ^{2} \alpha\right) \mathcal{Q}(\tan \alpha) .
\end{aligned}
$$

Equations (57), (59), (60), and (61) together constitute relations expressing the fractional operators in terms of their ordinary counterparts. Again, these can be used to realize fractional chirp multiplication and convolution operators in terms of their conventional counterparts. Since conventional chirp multiplication and chirp convolution correspond to lenses and sections of free space in optics, these formulas can be used to realize optical systems acting as fractional chirp multipliers or convolvers. Fractional chirp multipliers and convolvers can also be referred to as fractional lenses and fractional sections of free space.

By inserting Eq. (17) into Eq. (49) and using Eq. (57), we can also write the corresponding hyperdifferential Baker-Campbell-Hausdorff formula,

$$
\begin{aligned}
\exp \left\{-i \pi q\left[\cos ^{2} \alpha \mathcal{U}^{2}+\right.\right. & \sin \alpha \cos \alpha(\mathcal{U D}+\mathcal{D} \mathcal{U}) \\
\left.\left.+\sin ^{2} \alpha \mathcal{D}^{2}\right]\right\}= & \exp \left(i \pi \tan \alpha \mathcal{D}^{2}\right) \exp \left(-i \pi q \cos ^{2} \alpha \mathcal{U}^{2}\right) \\
& \times \exp \left(-i \pi \tan \alpha \mathcal{D}^{2}\right)
\end{aligned}
$$

We can use exactly the same technique on the remaining three Eqs. (59), (60), and (61) to obtain three more hyperdifferential Baker-Campbell-Hausdorff formulas as

$$
\begin{aligned}
\exp \left\{-i \pi q\left[\cos ^{2} \alpha \mathcal{U}^{2}+\right.\right. & \sin \alpha \cos \alpha(\mathcal{U D}+\mathcal{D} \mathcal{U}) \\
\left.\left.+\sin ^{2} \alpha \mathcal{D}^{2}\right]\right\}= & \exp \left(-i \pi \cot \alpha \mathcal{U}^{2}\right) \exp \left(-i \pi q \sin ^{2} \alpha \mathcal{D}^{2}\right) \\
& \times \exp \left(i \pi \cot \alpha \mathcal{U}^{2}\right), \\
\exp \left\{-i \pi q\left[\sin ^{2} \alpha \mathcal{U}^{2}-\right.\right. & \sin \alpha \cos \alpha(\mathcal{U} \mathcal{D}+\mathcal{D} \mathcal{U}) \\
\left.\left.+\cos ^{2} \alpha \mathcal{D}^{2}\right]\right\}= & \exp \left(-i \pi \cot \alpha \mathcal{D}^{2}\right) \exp \left(-i \pi q \sin ^{2} \alpha \mathcal{U}^{2}\right) \\
& \times \exp \left(i \pi \cot \alpha \mathcal{D}^{2}\right), \\
\exp \left\{-i \pi q\left[\sin ^{2} \alpha \mathcal{U}^{2}-\right.\right. & \sin \alpha \cos \alpha(\mathcal{U} \mathcal{D}+\mathcal{D} \mathcal{U}) \\
\left.\left.+\cos ^{2} \alpha \mathcal{D}^{2}\right]\right\}= & \exp \left(i \pi \tan \alpha \mathcal{U}^{2}\right) \exp \left(-i \pi q \cos ^{2} \alpha \mathcal{D}^{2}\right) \\
& \times \exp \left(-i \pi \tan \alpha \mathcal{U}^{2}\right) .
\end{aligned}
$$

\section{FRACTIONAL SCALING OPERATOR REVISITED}

We now discuss an interesting property of the fractional scaling operator:

Every linear canonical transform can be expressed as a fractional scaling operator with properly chosen quadratic phase factors at the input and output.

Since optical systems consisting of arbitrary concatenations of any number of sections of free space and lenses can be mathematically expressed as linear canonical transforms, this means that all such optical systems can be interpreted as a fractional scaling operator with properly chosen spherical reference surfaces at the input and output. This result can be expressed in terms of matrices as follows:

$$
\begin{aligned}
& {\left[\begin{array}{ll}
A & B \\
C & D
\end{array}\right]} \\
& =\left[\begin{array}{cc}
1 & 0 \\
\frac{1}{\lambda R_{2}} & 1
\end{array}\right]\left[\begin{array}{ll}
A^{\prime} & B^{\prime} \\
C^{\prime} & D^{\prime}
\end{array}\right]\left[\begin{array}{cc}
1 & 0 \\
\frac{-1}{\lambda R_{1}} & 1
\end{array}\right] \\
& =\left[\begin{array}{c}
A^{\prime}-\frac{B^{\prime}}{\lambda R_{1}} \\
\frac{A^{\prime}}{\lambda R_{2}}-\frac{D^{\prime}}{\lambda R_{1}}+B^{\prime}\left(1-\frac{1}{\lambda^{2} R_{1} R_{2}}\right)
\end{array} \frac{B^{\prime}}{\lambda R_{2}}+D^{\prime}\right],
\end{aligned}
$$

where $A D-B C=1 ; A^{\prime}, B^{\prime}, C^{\prime}, D^{\prime}$ are the matrix elements of the fractional scaling operator given in Eq. (40) $\left(B^{\prime}=C^{\prime}\right.$ ); and $R_{1}$ and $R_{2}$ are the radii of the spherical reference surfaces. The first and third matrices that appear in the right-hand side of Eq. (66), corresponding to multiplication by a quadratic-phase factor (chirp), account for the effect of using spherical reference surfaces. The solution for this system of equations for $B \neq 0$ is as follows:

$$
a=\frac{1}{\pi} \sin ^{-1}\left(\frac{2 B}{M-1 / M}\right)
$$




$$
\begin{aligned}
& R_{1}=\frac{B}{\lambda\left(M \cos ^{2} \alpha+\sin ^{2} \alpha / M-A\right)}, \\
& R_{2}=\frac{-B}{\lambda\left(M \sin ^{2} \alpha+\cos ^{2} \alpha / M-D\right)} .
\end{aligned}
$$

These equations mean that given $A, B, C, D$, we can choose either $M$ or $a$ but we cannot choose both of them independently. Once we choose either from Eq. (67), the other is determined, and the radii can be found by using Eqs. (68) and (69). Alternatively, we can start by choosing either $R_{1}$ or $R_{2}$. The resulting equation together with Eq. (67) will determine both $M$ and $a$ and also the other radius. In other words, given an arbitrary optical system consisting of lenses separated by sections of free space, we are able to interpret it as a fractional scaling operation provided that we choose $M, a$, and the two spherical reference surface radii as required by these equations. Notice that when $B=0, a$ takes on integer values (for $M \neq 1$ ), and the fractional imaging system turns into a conventional imaging system. The $M=1$ case merely corresponds to the identity operator and is of no interest to us.

In certain situations, one might not wish to have a spherical reference surface at the input (or the output). This case can be handled by simply putting $R_{1}=\infty$ (or $R_{2}=\infty$ ). In this case, $a, M$, and the other radius are fully determined in terms of the $A B C D$ parameters. The same would hold true if we were to impose a parametric constraint between $R_{1}$ and $R_{2}$, which effectively reduces them to a single parameter. This would be the case if we wished to evenly distribute the additional quadraticphase factors between the input and the output.

Note that if we wish to avoid complex values of $a$, we must choose $M$ such that $|B|<|M-1 / M| / 2$. This and the form of Eqs. (68) and (69) would also bound our freedom in those cases where we wish to specify $R_{1}$ or $R_{2}$ (or a constraint between them).

As an example, let us consider the well-known classical single-lens imaging system with distance $d_{1}$ from the object plane to the lens and $d_{2}$ from the lens to the image plane. The focal length of the lens is $f$. The above set of Eqs. (67)-(69) can be rewritten for this system as follows:

$$
\begin{aligned}
a & =\frac{1}{\pi} \sin ^{-1}\left[\frac{2 \lambda\left(d_{1}+d_{2}-d_{1} d_{2} / f\right)}{M-1 / M}\right], \\
R_{1} & =\frac{d_{1}+d_{2}-d_{1} d_{2} / f}{M \cos ^{2} \alpha+\sin ^{2} \alpha / M-1+d_{2} / f} \\
R_{2} & =\frac{-d_{1}-d_{2}+d_{1} d_{2} / f}{M \sin ^{2} \alpha+\cos ^{2} \alpha / M-1+d_{1} / f} .
\end{aligned}
$$

When the imaging condition $1 / f=1 / d_{1}+1 / d_{2}$ is satisfied, it is possible to show that $B=0$ and hence $a$ becomes an integer. If the parameters are such that we depart from this condition, that is, if the imaging condition is not exactly satisfied, then $a$ will assume a fractional value. In fact, this remains true not only for small deviations but for large ones as well. This supports referring to such systems as fractional imaging systems, as opposed to conventional "whole" imaging systems.

Using well-known matrix multiplication-based decomposition formulas given in Ref. 1 and several earlier studies, ${ }^{31,42,51}$ we can write

$$
\begin{aligned}
\mathcal{M}_{a}(M) & =\mathcal{Q}\left(\frac{1-D^{\prime}}{B^{\prime}}\right) \mathcal{R}\left(B^{\prime}\right) \mathcal{Q}\left(\frac{1-A^{\prime}}{B^{\prime}}\right) \\
& =\mathcal{R}\left(\frac{A^{\prime}-1}{B^{\prime}}\right) \mathcal{Q}\left(-B^{\prime}\right) \mathcal{R}\left(\frac{D^{\prime}-1}{B^{\prime}}\right),
\end{aligned}
$$

where $A^{\prime}, B^{\prime}, D^{\prime}$ are the matrix elements of the fractional scaling operator given in Eq. (40) $\left(C^{\prime}=B^{\prime}\right)$. Equating these equations to the expression obtained by substituting Eqs. (17) and (18) into Eq. (37) and expressing everything in terms of $\mathcal{U}$ and $\mathcal{D}$, we obtain hyperdifferential Baker-Campbell-Hausdorff formulas for $\mathcal{M}_{a}(M)$. Since these formulas are rather complicated, they are not explicitly presented.

It is also interesting to note that since the right-hand sides of Eqs. (73) and (74) consist only of chirp multiplication and chirp convolution operators, these equations suggest a way of implementing fractional scaling operators by use of sections of free space and thin lenses.

\section{DISCUSSION AND CONCLUSIONS}

Two operators are Fourier duals if what one operator does in the space domain corresponds to what the other one does in the frequency domain. Coordinate multiplication/differentiation and phase-shift/translation operators are common examples. In this paper we have considered and consolidated the continuum extension of this duality by employing the fractional Fourier transform and the concept of fractional Fourier domains. In other words, we have extended common dual pairs of operators to a continuum of operators that assume the common dual pair as special cases at opposite extremes.

In this paper we defined the fractional chirp multiplication/convolution and the fractional scaling operators. We expressed these operators in terms of their nonfractional special cases, revealing in what way they are combinations or interpolations of the common nonfractional operators. The results obtained are summarized in Table 1 along with corresponding results for coordinate multiplication/differentiation and phase-shift/translation operators that were previously established.

Of particular interest was the fractional scaling operator. Since mathematical scaling corresponds to optical imaging, we can also refer to this as the fractional imaging operator. We have seen that any optical system composed of lenses and sections of free space can be interpreted as a fractional scaling or imaging operator with spherical reference surfaces at the input and output. In other words, such optical systems, which do not satisfy the imaging condition, can be interpreted as fractional, rather than full, imaging systems. The fractional order of such imaging systems is a function of the parameters of the optical system. 
Table 1. Summary of Fractional Operators

\begin{tabular}{|c|c|c|}
\hline Fractional Operator & Symbol & Equivalent Expression \\
\hline $\begin{array}{l}\text { Fractional coordinate } \\
\text { multiplication }\end{array}$ & $\mathcal{U}_{a}$ & $\cos \alpha \mathcal{U}+\sin \alpha \mathcal{D}$ \\
\hline Fractional differentiation & $\mathcal{D}_{a}$ & $-\sin \alpha \mathcal{U}+\cos \alpha \mathcal{D}$ \\
\hline Fractional phase-shift & $\mathcal{P} \mathcal{H}_{a}(\xi)$ & $\exp \left(i \pi \xi^{2} \sin \alpha \cos \alpha\right) \mathcal{P H}(\xi \cos \alpha) \mathcal{S H}(\xi \sin \alpha)$ \\
\hline Fractional translation & $\mathcal{S H} \mathcal{H}_{a}(\xi)$ & $\exp \left(i \pi \xi^{2} \sin \alpha \cos \alpha\right) \mathcal{S H}(\xi \cos \alpha) \mathcal{P H}(-\xi \sin \alpha)$ \\
\hline Fractional scaling & $\mathcal{M}_{a}(M)$ & $\mathcal{F}^{-a} \mathcal{M}(M) \mathcal{F}^{a}, \mathcal{F}^{-1-a} \mathcal{M}(1 / M) \mathcal{F}^{1+a}$ \\
\hline Fractional chirp multiplication & $\mathcal{Q}_{a}(q)$ & $\mathcal{R}(-\tan \alpha) \mathcal{Q}\left(q \cos ^{2} \alpha\right) \mathcal{R}(\tan \alpha)$ \\
\hline Fractional chirp convolution & $\mathcal{R}_{a}(r)$ & $\mathcal{Q}(-\tan \alpha) \mathcal{R}\left(r \cos ^{2} \alpha\right) \mathcal{Q}(\tan \alpha)$ \\
\hline
\end{tabular}

The fractional chirp multiplication and the chirp convolution can be optically interpreted as fractional lenses and fractional sections, respectively, of free space. We have seen how these or fractional imaging systems can be realized in terms of common physical lenses and sections of free space. Although not emphasized in this paper, the fractional phase-shift operator can be likewise interpreted as a fractional prism, and the fractional translation operator can be interpreted as a fractional lateral translation of the optical axis. The formulas that we have provided also show how these can be realized in terms of common physical prisms and simple lateral physical translations of the optical axis.

Numerous applications require the frequency content of an optical signal or field to be altered. In such cases it is valuable to know the equivalent action to be taken in the space domain. Similarly, it has been shown that in certain cases improved performance or lower cost can be achieved by altering the content of the signal in a fractional domain, on the ath domain representation of a signal, rather than in the ordinary Fourier domain. An important use of the equations presented in this paper, expressing the fractional operators in terms of their nonfractional counterparts, is that these formulas are useful for implementing fractional operators with only the use of common physically available components.

\section{ACKNOWLEDGMENTS}

H. M. Ozaktas acknowledges partial support of the Turkish Academy of Sciences.

The authors' e-mail addresses are as follows:

U. Sümbül, uygar@alumni.bilkent.edu.tr; H. Ozaktas, haldun@ee.bilkent.edu.tr.

\section{REFERENCES AND NOTES}

1. H. M. Ozaktas, Z. Zalevsky, and M. A. Kutay, The Fractional Fourier Transform with Applications in Optics and Signal Processing (Wiley, New York, 2001).

2. D. Mendlovic and H. M. Ozaktas, "Fractional Fourier transforms and their optical implementation: I," J. Opt. Soc. Am. A 10, 1875-1881 (1993).

3. H. M. Ozaktas and D. Mendlovic, "Fractional Fourier transforms and their optical implementation: II," J. Opt. Soc. Am. A 10, 2522-2531 (1993).

4. H. M. Ozaktas and D. Mendlovic, "Fourier transforms of fractional order and their optical interpretation," Opt. Commun. 101, 163-169 (1993).
5. S. Abe and J. T. Sheridan, "Random fractional Fourier transform: stochastic perturbations along the axis of propagation," J. Opt. Soc. Am. A 16, 1986-1991 (1999).

6. O. Akay and G. F. Boudreaux-Bartels, "Unitary and Hermitian fractional operators and their relation to the fractional Fourier transform," IEEE Signal Process. Lett. 5, 312-314 (1998).

7. T. Alieva and M. J. Bastiaans, "Wigner distribution and fractional Fourier transform for two-dimensional symmetric optical beams," J. Opt. Soc. Am. A 17, 2319-2323 (2000).

8. L. M. Bernardo, "Independent adjustment of the scale and the order of polychromatic fractional Fourier transforms," Opt. Commun. 176, 61-64 (2000).

9. H. M. Ozaktas, B. Barshan, D. Mendlovic, and H. Urey, "Space-variant filtering in fractional Fourier domains," in Optical Computing, Institute of Physics Conference Series, B. S. Wherrett and P. Chavel, eds. (Institute of Physics, Bristol, UK, 1995), pp. 285-288.

10. M. A. Kutay, H. Ozaktaş, H. M. Ozaktas, and O. Arıkan, "The fractional Fourier domain decomposition," Signal Process. 77, 105-109 (1999).

11. İ. Ş. Yetik, M. A. Kutay, H. Özaktas, and H. M. Ozaktas, "Continuous and discrete fractional Fourier domain decomposition," in Proceedings of IEEE International Conference on Acoustics, Speech, and Signal Processing (Institute of Electrical and Electronics Engineers, New York, 2000), Vol. I, pp. 93-96.

12. İ. Ş. Yetik, H. M. Ozaktas, B. Barshan, and L. Onural, "Perspective projections in the space-frequency plane and fractional Fourier transforms," J. Opt. Soc. Am. A 17, 2382 2390 (2000).

13. L. Barker, C. Candan, T. Hakioğlu, M. A. Kutay, and H. M. Ozaktas, "The discrete harmonic oscillator, Harper's equation, and the discrete fractional Fourier transform," J. Phys. A 33, 2209-2222 (2000).

14. X. Deng, Y. Li, Y. Qiu, and D. Fan, "Diffraction interpreted through fractional Fourier transforms," Opt. Commun. 131, 241-245 (1996).

15. D. Dragoman, M. Dragoman, and K. H. Brenner, "Experimental demonstration of a continuously variant fractional Fourier transformer," Appl. Opt. 38, 4985-4989 (1999).

16. J. Hua, L. Liu, and G. Li, "Performing the fractional Fourier transform by one Fresnel diffraction and one lens," Opt. Commun. 137, 11-12 (1997).

17. M. A. Kutay and H. M. Ozaktas, "Optimal image restoration with the fractional Fourier transform," J. Opt. Soc. Am. A 15, 825-833 (1998).

18. S. T. Liu, L. Yu, and B. H. Zhu, "Optical image encryption by cascaded fractional Fourier transforms with random phase filtering," Opt. Commun. 187, 57-63 (2001).

19. S. C. Pei and J. J. Ding, "Two-dimensional affine generalized fractional Fourier transform,” J. Opt. Soc. Am. A 17, 2319-2323 (2000).

20. J. T. Sheridan and R. Patten, "Holographic interferometry and the fractional Fourier transformation," Opt. Lett. 25, $448-450$ (2000). 
21. R. Simon and K. B. Wolf, "Fractional Fourier transforms in two dimensions," J. Opt. Soc. Am. A 17, 2368-2381 (2000).

22. Z. Zalevsky, D. Mendlovic, M. A. Kutay, H. M. Ozaktas, and J. Solomon, "Improved acoustic signals discrimination using fractional Fourier transform based phase-space representations," Opt. Commun. 190, 95-101 (2001).

23. Y. Zhang, B.-Z. Dong, B.-Y. Gu, and G.-Z. Yang, "Beam shaping in the fractional Fourier transform domain," J. Opt. Soc. Am. A 15, 1114-1120 (1998).

24. D. M. Zhao and S. M. Wang, "Effect of misalignment on optical fractional Fourier transforming systems," Opt. Commun. 198, 281-286 (2001).

25. H. M. Ozaktas and D. Mendlovic, "Fractional Fourier optics," J. Opt. Soc. Am. A 12, 743-751 (1995).

26. H. M. Ozaktas and M. F. Erden, "Relationships among ray optical, Gaussian beam, and fractional Fourier transform descriptions of first-order optical systems," Opt. Commun. 143, 75-86 (1997).

27. H. M. Ozaktas and O. Aytür, "Fractional Fourier domains," Signal Process. 46, 119-124 (1995).

28. O. Aytür and H. M. Ozaktas, "Non-orthogonal domains in phase space of quantum optics and their relation to fractional Fourier transforms," Opt. Commun. 120, 166-170 (1995).

29. A. Papoulis, Systems and Transformations with Applications in Optics (McGraw-Hill, New York, 1968).

30. A. Lohmann, "Ein neues Dualitätsprinzip in der Optik," Optik 11, 478-488 (1954). An English version appeared as "Duality in optics," Optik 89, 93-97 (1992).

31. K. B. Wolf, Integral Transforms in Science and Engineering (Plenum, New York, 1979).

32. R. M. Wilcox, "Exponential operators and parameter differentiation in quantum physics," J. Math. Phys. 8, 962-982 (1967).

33. M. Moshinsky and C. Quesne, "Linear canonical transformations and their unitary representations," J. Math. Phys. 12, 1772-1780 (1971).

34. K. B. Wolf, "Canonical transforms. I. Complex linear transforms," J. Math. Phys. 15, 1295-1301 (1974).

35. R. Gilmore, "Baker-Campbell-Hausdorff formulas," J. Math. Phys. 15, 2090-2092 (1974)
36. A. J. Dragt and J. M. Finn, "Lie series and invariant functions for analytic symplectic maps," J. Math. Phys. 17, 2215-2227 (1976).

37. H. J. Butterweck, "General theory of linear, coherent, optical data-processing systems," J. Opt. Soc. Am. 67, 60-70 (1977).

38. J. Shamir, "Cylindrical lens systems described by operator algebra,” Appl. Opt. 18, 4195-4202 (1979).

39. M. J. Bastiaans, "Wigner distribution function and its application to first-order optics," J. Opt. Soc. Am. 69, 1710 1716 (1979).

40. M. Nazarathy and J. Shamir, "Fourier optics described by operator algebra," J. Opt. Soc. Am. 70, 150-159 (1980).

41. D. Stoler, "Operator methods in physical optics," J. Opt. Soc. Am. 71, 334-341 (1981).

42. M. Nazarathy and J. Shamir, "First-order optics-a canonical operator representation: lossless systems," J. Opt. Soc. Am. 72, 356-364 (1982).

43. M. Nazarathy and J. Shamir, "First-order optics—operator representation for systems with loss or gain," J. Opt. Soc. Am. 72, 1398-1408 (1982).

44. D. Basu and K. B. Wolf, "The unitary irreducible representations of $\mathrm{SL}(2, R)$ in all subgroup reductions," J. Math. Phys. 23, 189-205 (1982).

45. M. Kauderer, "Fourier-optics approach to the symplectic group," J. Opt. Soc. Am. A 7, 231-239 (1990).

46. M. Kauderer, Symplectic Matrices: First Order Systems and Special Relativity (World Scientific, Singapore, 1994).

47. R. Simon and K. B. Wolf, "Structure of the set of paraxial optical systems," J. Opt. Soc. Am. A 17, 342-355 (2000).

48. H. M. Ozaktas, B. Barshan, D. Mendlovic, and L. Onural, "Convolution, filtering, and multiplexing in fractional Fourier domains and their relation to chirp and wavelet transforms," J. Opt. Soc. Am. A 11, 547-559 (1994).

49. It should be noted, however, that the fractional power of an operator is not unique. For instance, see Ref. 21 or Ref. 1 , pp. 137-143.

50. C. Cohen-Tannoudji, B. Diu, and F. Laloë, Quantum Me chanics (Wiley, New York, 1977).

51. A. Papoulis, Signal Analysis (McGraw-Hill, New York, 1977). 